\title{
Conformational Flexibility Enables Function of a BECN1 Region Essential for Starvation-Mediated Autophagy
}

\author{
Yang Mei $^{\mathrm{a}}$, Arvind Ramanathan ${ }^{\mathrm{b}}$, Karen Glover $^{\mathrm{a}}$, Christopher Stanley ${ }^{\mathrm{c}}$, Ruslan Sanishvilid, \\ Srinivas Chakravarthy ${ }^{\mathrm{e}}$, Zhongyu Yang ${ }^{\mathrm{a}}$, Christopher L. Colbert ${ }^{\mathrm{a}}$, and Sangita Sinha ${ }^{\mathrm{a},{ }^{*}}$ \\ aDepartment of Chemistry and Biochemistry, North Dakota State University, Fargo, ND \\ 58108-6050, USA \\ bHealth Data Sciences Institute, Computational Science and Engineering Division, Oak Ridge \\ National Laboratory, Oak Ridge, TN 37830, USA \\ 'Biology and Soft Matter Division, Oak Ridge National Laboratory, Oak Ridge, TN 37831, USA \\ dGMCA@APS, X-ray Science Division, Advanced Photon Source, Argonne National Laboratory, \\ Argonne, IL 60439, USA \\ eBio-CAT, Advanced Photon Source, 9700 South Cass Avenue, Bldg. 435B, Argonne, IL 60439, \\ USA
}

\begin{abstract}
BECN1 is essential for autophagy, a critical eukaryotic cellular homeostasis pathway. Here we delineate a highly conserved BECN1 domain located between previously characterized BH3 and coiled-coil domains, and elucidate its structure and role in autophagy. The 2.0 A Sulfur-SAD Xray crystal structure of this domain demonstrates that its $\mathrm{N}$-terminal half is unstructured, while its C-terminal half is helical, hence we name it the Flexible Helical Domain (FHD). Circular dichroism spectroscopy, Double-Electron-Electron Resonance-Electron Paramagnetic Resonance and Small Angle X-ray Scattering (SAXS) analyses confirm that the FHD is partially disordered, even in the context of adjacent BECN1 domains. Molecular dynamic simulations fitted to SAXS data indicate that the FHD transiently samples more helical conformations. FHD helicity increases in 2,2,2-trifluoroethanol suggesting it may become more helical upon binding. Lastly, cellular studies show that conserved FHD residues are required for starvation-induced autophagy. Thus, the FHD likely undergoes a binding-associated disorder-to-helix transition and conserved residues critical for this interaction are essential for starvation-induced autophagy.
\end{abstract}

\section{Graphical abstract}

\footnotetext{
*To whom correspondence should be addressed: ; Email: sangita.sinha@ndsu.edu. SUPPORTING INFORMATION AVAILABLE

Table S1. Comparison of molecular parameters calculated from SAXS data and the X-ray crystal structure.

Table S2. Summary of MD simulation parameters for the FHD system.

Table S3. Secondary structure content of FHD-containing constructs in different TFE concentrations.

Figure S1. CD spectra of different BECN1 constructs.

Figure S2. The FHD crystal structure fits poorly to the SAXS data.

Figure S3. SAXS analysis of BECN1 CCD.

Figure S4. Pseudo-atomic model of the BECN1 FHD-CCD.
} 


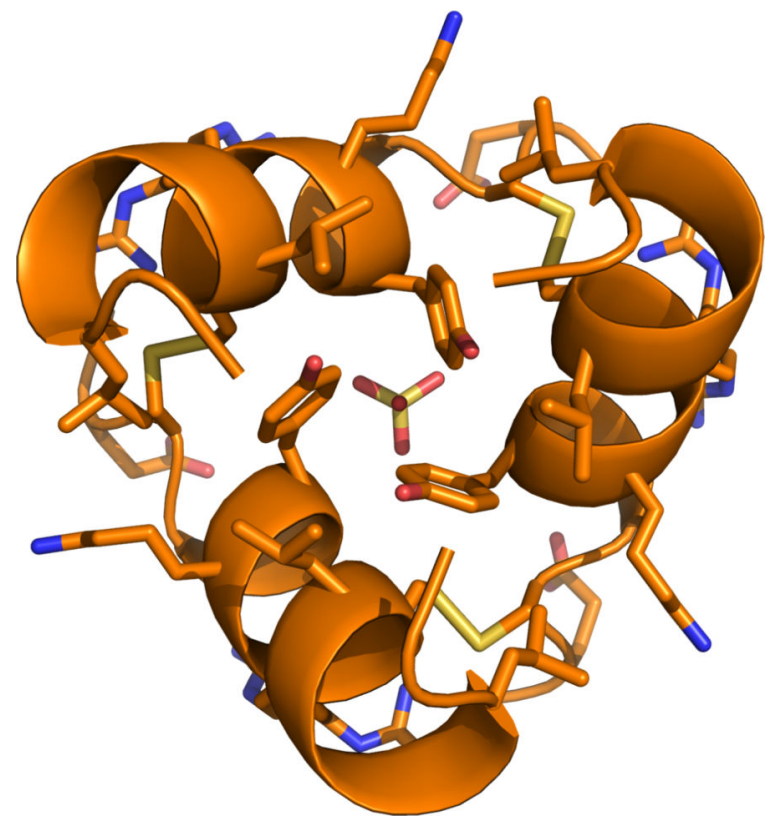

\section{Keywords}

ECN1; Autophagy; Intrinsically disordered region; Structural transition; Flexible Helical Domain

BECN1 (also called Beclin 1, ATG6 or VPS30), a protein conserved in all eukaryotes, is required for macroautophagy. Macroautophagy (herewith referred to as autophagy) is a conserved catabolic homeostasis cellular pathway, during which, targeted cytoplasmic constituents such as long-lived proteins, damaged organelles and pathogens are engulfed by multi-layered vesicles to form autophagosomes that subsequently fuse with lysosomes, enabling degradation of the engulfed materials (1-3). As autophagy facilitates recycling of surplus, damaged or harmful macromolecular assemblies and provides a source of nutrients in starvation conditions, it plays a role in many physiological processes. Consequently, aberrant or reduced autophagy, such as that caused by defective or altered levels of BECN1, is implicated in numerous diseases including cancers, cardiovascular diseases, muscular and neurodegenerative disorders, and several infectious diseases; while increased autophagy such as due to caloric restriction is implicated in cell survival, longer life span, reduction of aggregates implicated in neurological diseases and better cellular innate defenses (4-7).

BECN1 is required for autophagosome nucleation and is an essential component of the Class III Phosphotidylinositol-3-kinase (PI3KC3) complex. In addition to PI3KC3 and BECN1, this complex also contains other key autophagy proteins: VPS15 (p150), a Ser/Thr kinase adaptor protein; and either ATG14 (BARKOR) or VPS38 (UVRAG) (8-11). Besides autophagosome nucleation, this complex plays important roles in others stages of autophagy, vesicle trafficking pathways and vacuolar sorting $(3,9,12)$. The function of this core complex is modulated via interactions with numerous autophagy regulators, with many of these interactions being mediated via BECN1 (13-16). Indeed, BECN1 is implicated in binding to approximately twenty cellular proteins as well many virus-encoded proteins (17- 
20). In addition to these functions, BECN1 has been shown to localize to the nucleus (21). The nuclear function of BECN1 is unclear although it may play a role in nuclear export (2123) and/or transcriptional regulation of autophagy proteins (22). BECN1 has been established as a locus of coordination for the cellular pathways of autophagy and apoptosis $(15,17,24)$, as the anti-apoptotic BCL2 proteins bind to BECN1 and down-regulate autophagy $(25,26)$. More recently, it was shown that caspase-cleaved proteolytic BECN1 fragments are ineffective in autophagy and instead facilitate apoptosis $(27,28)$.

Structural and biophysical studies of individual BECN1 domains have provided important information regarding the structure and conformational flexibility of this complex protein (29-35), laying the groundwork for understanding the molecular mechanism by which BECN1 interacts with its numerous, diverse partners and mediates autophagy. Based on structural studies, three domains have been delineated in the 450-residue human BECN1: (1) an intrinsically disordered region (IDR, comprising residues 42-130) (16); (2) a coiledcoiled domain (CCD, residues 175-265) (33); and (3) a $\beta$-a-repeat autophagy-specific domain (BARAD, residues $248-450)(34,35)$. While the BECN1 IDR is poorly conserved amongst homologs, BECN1 residues 136-450, are highly conserved, hence, the C-terminal part of this region is sometimes also called the evolutionarily-conserved domain (ECD) (34, 36). Residues 105-130 within the IDR constitute a BCL2 Homology 3 domain (BH3D), a functional domain necessary and sufficient for binding to diverse BCL2 homologs $(26,37$, $38)$.

Despite the extensive research focus on BECN1, the structure of full-length BECN1 has not yet been determined because the conformational flexibility of BECN1 prevents analysis using canonical structural methods. Recent cryo-EM reconstructions of the complex comprising BECN1, PI3KC3, p150, and either ATG14 or UVRAG indicates that the complex has a V-shaped molecular envelope and outlines relative arrangements of the different proteins (39). The BECN1 BARAD was modeled at the tip of one arm of the Venvelope, while the $\mathrm{N}$-terminus was positioned at the base of the V-envelope, but it was not possible to elucidate structural details of BECN1, or of its interactions with other components of this complex. Hence, many unanswered questions remain regarding BECN1 structure, interactions and mechanism.

Residues 141-171 located between the BH3D and the CCD were previously predicted to be part of the CCD (40), but structural delineation of the boundaries of the BH3D (26) and CCD (33) indicate that these residues comprise an independent domain. Here we report our structural, biophysical and functional studies characterizing the human BECN1 domain between the BH3D and CCD. We have used various methods: X-ray crystallography, Circular Dichroism (CD) spectroscopy, Double Electron-Electron Resonance (DEER)Electron-Paramagnetic Resonance (EPR) spectroscopy and Small-Angle X-ray Scattering (SAXS) to demonstrate that, while the C-terminal half of this domain forms an a-helix, the $\mathrm{N}$-terminal half is disordered in the absence of a binding partner. Further, we find that this disorder persists even in larger BECN1 constructs containing the adjacent BH3D and CCD, suggesting that this domain is partially disordered even in the context of the full-length protein. Therefore, we name residues 141-171 the flexible helical domain (FHD). Interestingly, the FHD crystal structure and SAXS data indicate that the BECN1 FHD 
trimerizes in the absence of a binding partner. In order to better understand FHD conformations in the absence of binding partners we performed long time-scale molecular dynamic (MD) simulations, which based on fits of different conformers to the experimental SAXS data, also suggests that the FHD appears to exist as a trimer, wherein each FHD has a helical C-terminal part and an unstructured $\mathrm{N}$-terminal region that transiently samples helical conformations. Highly conserved FHD residues map to one face of the helical part of the FHD, suggesting that this domain may be involved in binding. Additionally, we find that the FHD is predicted to fold upon binding, and show that this region undergoes environment-influenced disorder-to-helix transitions. Lastly, and most importantly, we show that conserved FHD residues are critical for starvation-triggered up-regulation of autophagy. In sum, our results indicate that the BECN1 flexible helical domain (FHD) comprising residues $141-171$ is partially disordered, but likely undergoes a disorder-to-helix transition positioning all conserved residues in this region on one face of the helix, which is essential for binding to appropriate protein partners, and for the starvation-induced up-regulation of autophagy.

\section{EXPERIMENTAL PROCEDURES}

\section{Production of different BECN1 Protein Fragments}

The human BECN1 FHD (residues 141-171) (Figure 1) was chemically synthesized, HPLC purified to $>95 \%$ purity with purity confirmed by electrospray mass spectrometry (Protein Chem. Tech. Core, UTSW or EZBioLabs). A $1 \mathrm{mM}$ peptide stock solution in $25 \mathrm{mM}$ HEPES pH 7.5, $150 \mathrm{mM} \mathrm{NaCl}, 2 \mathrm{mM}$ b-mercaptoethanol was prepared. For EPR analysis, chemical synthesis was used to obtain an FHD construct that included a Cys preceding the FHD, while C159 was altered to a Ser. BECN1 CCD (residues 175-265), FHD-CCD (residues 141-265) and BH3D-FHD-CCD (residues 105-265) were cloned between the BamHI and NotI restriction enzyme sites of the pMBP-Parallel-1 vector (41). E. coli BL21(DE3)pLysS cells were transformed by expression vectors and grown in LB media supplemented with $100 \mu \mathrm{g} / \mathrm{mL}$ ampicillin at $37^{\circ} \mathrm{C}$ to an $\mathrm{OD}_{600}$ of $\sim 0.8$ prior to induction of recombinant protein expression by addition of $0.5 \mathrm{mM}$ isopropyl thio- $\beta$-D-galactoside (IPTG) at $20^{\circ} \mathrm{C}$ overnight. Soluble MBP-tagged fusion protein was purified from clarified crude cell lysate by amylose affinity chromatography. The MBP tag was removed by oncolumn tobacco etch virus (TEV) protease-cleavage. Subsequently, the protein was purified to homogeneity by size exclusion chromatography (SEC), using a 16/60 Superdex200 or 10/30 Tandem Superdex 200+75 (GE Lifesciences, Pittsburgh, US) columns. At each stage of purification, protein purity was evaluated by SDS-PAGE stained with Coomassie Blue. In each case, the final purified protein was estimated to be $>90 \%$ pure by Coomassie Blue stained SDS-PAGE.

\section{Sequence Analysis}

Sequences of BECN1 homologs from eight diverse eukaryotes: Homo sapiens, Mus musculus, Gallus gallus, Zenopus laevis, Drosophila melanogaster, Caenorhabditis elegans, Arabidopsis thaliana, and Saccharomyces cerevisiae, were identified by BLASTP searches of Genomic RefSeq Protein databases (http://blast.ncbi.nlm.nih.gov/) for each organism. Multiple sequence alignment of these orthologs was performed with Jalview (http:// 
www.jalview.org/). Disordered regions and Anchor regions within the FHD were predicted using the intrinsically unstructured protein predictor, IUPred, (http://upred.enzim.hu/), and ANCHOR (http://anchor.enzim.hu/) respectively (42-44).

\section{Crystallization, X-ray Diffraction Data Collection and Structure Solution}

BECN1 FHD crystals were grown at $20^{\circ} \mathrm{C}$ by sitting drop vapor diffusion from a drop consisting of $1 \mu \mathrm{l}$ of $10 \mathrm{mg} / \mathrm{m} 1$ FHDin $25 \mathrm{mM}$ HEPES, pH 7.5; $250 \mathrm{mM} \mathrm{NaCl} ; 2 \mathrm{mM} \mathrm{b}$ mercaptoethanol and $1 \mu \mathrm{l}$ of reservoir solution, comprising $100 \mathrm{mM}$ sodium citrate tribasic dihydrate, $\mathrm{pH} 5.6 ; 250 \mathrm{mM}$ potassium sodium tartrate tetrahydrate; and 2.2 $\mathrm{M}$ ammonium sulfate. Cubic crystals were observed within 4 days and harvested after $\sim$ two months. The crystals were cryo-protected with MiTeGen LV Cryo-oil (MiTeGen, Ithaca, NY) and immediately flash-cooled in liquid $\mathrm{N}_{2}$. Diffraction intensities from these crystals were recorded at $100 \mathrm{~K}$ using 1 second exposure per $0.5^{\circ}$ crystal rotation per image on a MARCCD 300 detector at a crystal-to-detector distance of $150 \mathrm{~mm}$ at beamline 23ID-D of GMCA@APS, ANL, Chicago. The data used to solve the structure were collected at an Xray wavelength of $1.3776 \AA$ in a $360^{\circ}$ sweep from a single crystal. Data were processed using XDS (45). Diffraction data statistics are summarized in Table 1. The structure was solved by S-SAD using SHELXD (46). The model was built using Coot (47) and refined in PHENIX (48) (Table 1). The final refined structure is deposited in the PDB under PDB ID 5EFM.

\section{Spectroscopy}

CD spectra were recorded at $4{ }^{\circ} \mathrm{C}$ between 195 and $250 \mathrm{~nm}$ using a $300 \mu \mathrm{L}$ quartz cell with a $0.1 \mathrm{~cm}$ path length on a Jasco J-810 spectropolarimeter equipped with thermoelectric temperature control. BECN1 constructs were diluted to 50-200 $\mu \mathrm{M}$ in CD buffer (10 mM potassium phosphate, $\mathrm{pH}$ 7.6; $100 \mathrm{mM}$ ammonium sulfate). 2,2,2-tetrafluoroethanol (TFE) (Sigma Aldrich, St. Louis, MO) was premixed and incubated with BECN1 constructs in CD buffer at different $\% \mathrm{v} / \mathrm{v}$ ratios: $0 \%, 10 \%, 25 \%$ or $40 \%$. The data were analyzed using SELCON3 from the CDpro program suite (49).

\section{DEER-EPR Data Collection and Analysis}

C140 preceding the FHD and C165 in the FHD domain were labeled with the (1oxyl-2,2,5,5-tetramethylpyrroline-3-methyl) methanethiosulfonate spin label (50). Unreacted spin label was removed by washing the peptide/label mixture through a $3 \mathrm{kDa}$-cutoff Millipore concentrator. The sample concentration for each EPR experiment was $\sim 200 \mu \mathrm{M}$, with $20 \%(\mathrm{v} / \mathrm{v})$ glycerol mixed in as a cryogenic protectant. Immediately prior to data collection, the sample was flash-cooled in quartz capillaries using a liquid nitrogen bath. All DEER-EPR measurements were performed on an Elexsys 580 spectrometer (Bruker Corp., Billerica, MA) equipped with a Super Q-FTu Bridge, a Bruker ER 5107DQ resonator and a $10 \mathrm{~W}$ Q-band signal amplifier. The Oxford temperature controller and liquid nitrogen were used to maintain the experimental temperature at $80 \mathrm{~K}$. A standard pulse sequence and operation procedure were used for DEER-EPR as described (51), with pulse lengths adjusted to 16 and $32 \mathrm{~ns}$ for a $\pi / 2$ and $\pi$ pulse, respectively. The time domain signal collected for each sample was about $3 \mu \mathrm{s}$. DEER-EPR data were analyzed using a model-free maximumentropy analysis approach by Christian Altenbach (http://www.chemistry.ucla.edu/directory/ 
hubbell-wayne-1). The reliable maximum average distance extracted from the DEER data,

$\mathrm{r}_{\max <\mathrm{r}>}$, can be estimated using $I_{\max ,<\mathrm{r}>} \approx 5 \times\left[t_{\max } /(2 \mathrm{~ms})\right]^{1 / 3} \mathrm{~nm}$, where $\mathrm{t}_{\max }$ is the maximum length of time domain signal (52). In our case, $r_{\max }<\mathrm{r}>$ is approximately $60 \AA$.

\section{SAXS Data Collection and Analysis}

SAXS data were recorded at the BioCAT beamline (ID18) (APS, Argonne, IL) on a Mar165$\mathrm{CCD}$ detector, at a sample to detector distance of $2.5 \mathrm{~m}$, thereby covering a momentum transfer range of $0.006 \AA^{-1}<q<0.32 \AA^{-1}$. SAXS measurements were recorded for BECN1 constructs smaller than 50 residues by loading a quartz capillary at various sample concentrations ( $4 \mathrm{mg} / \mathrm{ml}, 2 \mathrm{mg} / \mathrm{ml}, 1 \mathrm{mg} / \mathrm{ml}$, and $0.5 \mathrm{mg} / \mathrm{ml}$ ). For larger BECN1 constructs, we performed Size Exclusion Chromatography (SEC) in tandem with SAXS data collection, to ensure that the SAXS data was collected from a homogeneous sample. Four mg/ml protein was injected onto a SEC column (GL 10/300 Superdex 200) and SAXS data recorded by exposing the column eluate to the X-ray beam for 1 second with a periodicity of 5 seconds. Scattering data were normalized to the incident X-ray beam intensity and scattering from buffer was subtracted prior to analysis using Igor Pro macros (53). Data analysis was performed using the ATSAS program suite (54) (http://www.emblhamburg.de/ biosaxs/crysol.html). Within the ATSAS suite, the program PRIMUS (55) was used to calculate Guinier extrapolations to calculate the radius of gyration $\left(\mathrm{R}_{\mathrm{g}}\right)$ and Kratky plots to evaluate disorder within the sample. The program GNOM (56) was used to plot the $\mathrm{P}(r)$ function from the Fourier inversion of the scattering intensity, $I(\mathrm{q})$. The $P(\mathrm{r})$ function was used to calculate the $\mathrm{R}_{\mathrm{g}}$ and maximum particle size $\left(\mathrm{D}_{\max }\right)$, and also for the reconstruction of ab initio envelopes by the application of ten cycles in DAMMIF (57). The resulting bead models were sequentially analyzed using DAMSEL, DAMSUP, and DAMAVER(58) to compare and identify the most probable model, align all models to the most probable model, average these aligned models and compute a probability map with the averaged model then filtered using DAMFILT (58). CRYSOL (59) was used to compare theoretical scattering curves calculated for FHD monomer and trimer atomic structures against the experimental SAXS scattering curve. The FHD monomer and trimer structures were superimposed into the final bead models using the program SUPCOMB (60). For the FHD-CCD construct the crystal structures of the FHD and CCD were used in SASREF (61) to build and fit a model against the corresponding SAXS data sets. The four-residue gap between the FHD and CCD was set to $10 \AA$ (the average length between an a helical motif and fully extended motif with 4 residues), and P2 symmetry was imposed on all models.

\section{Simulations}

MD simulations were initiated from five different starting models. One model was comprised of the entire FHD monomer modeled as a disordered structure. In the other four models, the C-terminal end of the FHD was retained as a helix, as observed in the crystal structure. In two of these models, the FHD N-terminal residues 141-159 were modeled in a random coil conformation, while in the other pair, the $\mathrm{N}$-terminal residues were modeled as an a-helix such that the entire FHD was a single helix. The models were then placed in different oligomeric states: either a monomer or the trimer observed in the crystal structure. Long time-scale MD simulations were performed for each of the models. The simulations are summarized in Table S2. Briefly, the parm99SB.ildn force field in the AMBER 12.0 
suite of molecular modeling software was used, as it is particularly well suited for running long time-scale simulations. After modeling the structures using standard amino acid residues, and determining the protonation state for each residue at $\mathrm{pH}$ 7.0, each system was neutralized using counter-ions placed in a solvated box of TIP3P water molecules that was at least $12 \AA$ from the protein. Final box sizes and the total number of atoms in each simulation system are detailed in Table S2. Each system was minimized and equilibrated as previously described (62). Production runs were carried out using the 'constant number of particles, volume and energy ensemble with particle mesh Ewald' technique for electrostatics and a 10 $\AA$ A cut-off for Lennard-Jones interactions. The SHAKE algorithm was used to restrict motions of all covalently bound hydrogen atoms. Simulations were performed at $1 \mathrm{~atm}$ pressure and at $300 \mathrm{~K}$ temperature and the data from the simulations were stored every 0.02 ns. The simulations are summarized in Table S2.

\section{Autophagy Assays}

Cellular autophagy levels were evaluated by monitoring cellular localization of GFP-tagged LC3 (63). $5.0 \times 10^{5}$ MCF7 cells were seeded in each chamber of a 4-well culture slide (Millipore EZ slides) and cultured overnight in DMEM with 10\% FBS until 80\% confluency. The cells were co-transfected with a total of $4 \mu \mathrm{g}$ plasmids comprising $1.6 \mu \mathrm{g}$ GFP-LC3 and $2.4 \mu \mathrm{g}$ BECN1 WT or mutant expression plasmids, using lipofectamine 2000 (Invitrogen, Carlsbad, CA) according to the manufacturer's instructions. After transfection, the cells were cultured in either rich (DMEM, 10\% FBS, 2X amino acid mixture) or starvation (EBSS) media respectively for 4 hours. Cells were then fixed with $2 \%$ paraformaldehyde in PBS. GFP-LC3-positive puncta were observed under a fluorescent microscope (Zeiss AxioObserver Z1) and quantified by counting a minimum of 100 cells for duplicate samples per condition using Imaris (Bitplane AG, Zurich, Switzerland) in three independent repeats. The significance of alterations in autophagy levels was determined by a two-tailed, heteroscedastic student's t-test, wherein $\mathrm{p} \leq 0.05$ is considered significant.

\section{RESULTS}

\section{The FHD is Partially Disordered}

We attempted to crystallize several different constructs of human BECN1 that included the highly-conserved FHD (Figure 1), including the FHD in isolation, the BH3D-FHD, FHDCCD and BH3D-FHD-CCD; as well as full-length BECN1. Perhaps the inherent flexibility prevented crystallization of the larger multi-domain constructs of BECN1, however, we were successful in crystallizing the construct comprising residues 141-171. These crystals belonged to the space group $\mathrm{P}_{3} 32$ with unit cell parameters of $a=b=c=63.2 \AA$, and contained one copy of the BECN1 FHD per asymmetric unit (Table 1). The $2.0 \AA$ crystal structure of the FHD was determined using Sulfur SAD methods. To our surprise, electron density was visible only for residues 157-171, of which residues 160-169 form a 2.5-turn amphipathic a-helix (Figure 2A). Electron density corresponding to residues 141-156 is missing, suggesting that similar to the BH3D preceding it (16), this region may be unstructured in certain physiological contexts. Notably, the FHD was not identified as part of the consensus IDR delineated for BECN1, however, the programs IUPRED and Psipred predict that residues 141-152/154 are disordered. 
In the crystal structure, three FHD helices pack around the crystallographic threefold to form a compact trimer (Figure 2B). The core of the trimer is stabilized by aromatic stacking of the conserved Y162 residues from each helix, with each Y162 being largely buried in the hydrophobic interface (Figure 2B). Other hydrophobic residues from each of the three helices, including conserved residues L166 and L169, also contribute to the hydrophobic packing at the trimer center. The trimer is further stabilized by three disulfide bonds between C159 and C165 of adjacent FHD molecules (Figure 2B). The formation of the trimer buries about one-third of the total surface area of $1773 \AA^{2}$ of each monomer.

One face of this trimer is negatively charged, primarily due to the glutamate at the Cterminus of each helix (Figure 2C). The opposite trimer face is positively charged, with the outside edge of this positive face bearing residues K163 and R164 from each monomer, and the center caving into a positively charged pocket created by the helix dipoles and lined by the side chain amides of N161 and backbone amides of Y162 from each FHD. A sulfate from the crystallization solution is bound at the crystallographic three-fold within this pocket (Figure 2).

\section{The FHD is Partially Disordered in Solution, Even in the Context of Adjacent BECN1 Domains}

In order to confirm that the FHD was partly disordered in the context of full-length BECN1, we used CD spectroscopy to assess and compare the secondary structure content of BECN1 constructs containing the FHD and/or adjacent domains: FHD, BH3D, CCD, FHD-CCD, BH3D-FHD-CCD (Figure S1). Secondary structure content was quantified (Table 2) by analyzing these CD spectra using SELCON3 (64), which provides the most accurate estimation of disordered structure (65). As has been noted before, CD spectra of the BH3D has a negative peak at around $195 \mathrm{~nm}$ (Figure S1, cyan curve), and was estimated by SELCON3 to contain 24 residues in random coil conformation (Table 2), indicating that it is completely disordered without an interaction partner (16). In contrast, the CD spectra of the CCD (Figure S1, magenta curve) displays a positive transition at $\sim 195 \mathrm{~nm}$ and a large negative split transition at $208 \mathrm{~nm}$ and $222 \mathrm{~nm}$, typical of all-helical proteins. However, only 79 of a total of 97 residues were estimated to be in a helical conformation (Table 2), somewhat less than that modeled as a helix in the CCD crystal structure (33), and 19 residues were estimated to be in a coil-conformation. The CD spectrum of the FHD (Figure S1, orange curve), shows two negative peaks: a lesser peak at $\sim 222 \mathrm{~nm}$ which is typical of ahelices, and a larger peak at $\sim 205 \mathrm{~nm}$ which is an intermediate position between the negative transition typical of random coils and the first negative peak typical of a-helices. The secondary structure content estimated from this spectrum indicates that the FHD has 12 helical residues and 15-19 disordered or coil residues (Table 2), consistent with the FHD crystal structure described above.

The two-domain FHD-CCD has a spectrum (Figure S1, green trace) more similar to the helical CCD, but with smaller negative transitions at $208 \mathrm{~nm}$ and $222 \mathrm{~nm}$, and an estimated secondary structure content of 103 helical residues and 25 random coil residues. Relative to the sum of the secondary structure content estimated for the individual FHD and CCD, this corresponds to an additional ten helical residues, with an equivalent reduction in the number 
of residues in coil confirmation. This is likely due to an increase in the helicity of the CCD within the two-domain construct, to levels that better approximate the helicity of the CCD crystal structure.

Lastly, the BH3D-FHD-CCD construct (Figure S1, red trace) has a helical content of 105 residues, and a random coil content of 51-59 residues (Table 2), consistent with the sum of the secondary structure contents of the BH3D and the FHD-CCD constructs. This indicates that this three-domain construct contains a completely disordered BH3D, partially disordered FHD and helical CCD. In sum, these CD data indicate that the FHD does not become completely helical even when the flanking disordered BH3D and helical CCD domains are present.

\section{The FHD is an Extended, Significantly-Disordered Domain in Solution}

In order to better understand the size and conformation of the FHD in solution, we carried out residue-to-residue, intra-domain distance measurement using DEER-EPR spectroscopy (52) and Site-Directed Spin Labeling (50). Residue C159 within the FHD was substituted with Ser, while C140, a residue preceding the FHD, and C165 located toward the FHD Cterminus, were spin-labeled with 1-oxyl-2,2,5,5-tetramethylpyrroline-3-methyl) methanethiosulfonate to create the spin labeled mutant 140R1/165R1, where R1 represents the spin label side chain. We analyzed the time domain signals (Figure 3A) using a previously reported DEER data analysis program (51). Since we anticipated that the FHD is structurally disordered, we used a Gaussian function to describe the inter-spin distance distribution in the data analysis. The resultant distance distribution extracted from the DEER data (Figure 3B) displays a broad spin-spin distance distribution, ranging from $\sim 15$ to $55 \AA$, qualitatively consistent with an intrinsically disordered, extended FHD structure. The DEER data were also analyzed using a model-free, unbiased analysis, which assumes that every distance between 15 and $100 \AA$ contributes to the time domain signal, while the probability of each distance is plotted against the distance. Data analyzed by this approach also indicate an intrinsically disordered, extended FHD structure.

We used SAXS to further investigate the size, shape and structure of the BECN1 FHD in solution. The radius of gyration $\left(\mathrm{R}_{\mathrm{g}}\right)$ of the FHD, estimated from the Guinier plot and $\mathrm{P}(\mathrm{r})$ distribution, was $\sim 17.5 \AA$ (Figure $4 \mathrm{~A}, 4 \mathrm{~B}$ ), which is significantly larger than the $\mathrm{R}_{\mathrm{g}}$ of $10 \AA$ or $\sim 12 \AA$ for the FHD monomer or trimer respectively, calculated from the crystal structure (Table S1). Similarly, the $\mathrm{D}_{\max }$ obtained from the $\mathrm{P}(\mathrm{r})$ (Figure 4B and Table $\mathrm{S} 1$ ) is $61.4 \AA$, nearly triple the length of the crystal structure (Table S1). This is consistent with the presence of additional disordered residues that were not visible in the crystal structure and the partial disorder estimated from analysis of the $\mathrm{CD}$ spectra. This partial disorder is confirmed by the Kratky plot (Figure 4C). Lastly, neither the FHD monomer, nor the trimer crystal structures fit well ( $\chi^{2}=7.5$ and 6.5 respectively) into molecular envelopes calculated from the SAXS data, leaving a lot of unoccupied space within the envelope indicative of disordered regions that were not modeled (Table S1, Figure S2). 


\section{The FHD Appears to Transiently Sample More Helical Conformations}

The SAXS analyses of the FHD provided gross structural details of its solution structure. To further probe the conformational heterogeneity and oligomerization of the FHD in solution and enable atomistic characterization of the observed conformational disorder in FHD, we performed long time-scale all-atom MD simulations using five different FHD starting models (Table S2): (i) a completely disordered FHD monomer; (ii) a completely helical FHD monomer; (iii) a part coil and part helical FHD monomer (as per the crystal structure); (iv) completely helical FHD trimer; and (v) part coil and part helical FHD trimer (as per the crystal structure). The sulfate was not included in the trimer models used for simulation. We found that regardless of the starting model, the C-terminus of the FHD retained, or folded into its helical state. Further, although the $\mathrm{N}$-terminus tended to remain disordered, or unfold in simulations starting from models of a completely helical FHD; a majority of conformations sampled folded states during the course of the simulations.

We used a heuristic, similar to a recently developed approach (66), to assess the fit of the MD conformational ensembles from different starting models to the experimental SAXS data using CRYSOL (59). In general, the scattering curves calculated from the ensembles of MD simulated conformations generated from the various monomer models did not fit well (average $\chi^{2}$ of $\sim 7$ ), but the conformers generated from the trimer models fit the experimental SAXS curves quite well (average $\chi^{2}$ of 2.7). This suggests that the FHD tends to form a trimer in solution. Therefore, we focused subsequent analyses on the simulations starting from the two trimer models.

In order to obtain a better fit to the SAXS experimental scattering curves, for each trimer simulation, we selected conformers with low $\chi^{2}$ values (between 0.5 and 1.5) and reconstructed an ensemble representation of the FHD trimer. We find that both ensembles of conformers were needed to fit the experimental data (Figure 5). Further, the ensembles of conformations that have the best fits show considerable heterogeneity (Figure 5), including a high degree of conformational variation in residues 141-151, with many of the conformations showing partial helices. These results strongly suggest that the FHD has an inherent propensity to interconvert between disorder and helical conformations.

\section{The FHD-CCD Adopts an Extended Tertiary Structure}

Next we used SEC-SAXS in order to obtain a better understanding of the size, shape and structure of the BECN1 FHD-CCD in solution. SEC-SAXS data were recorded from the CCD, as well as the two-domain FHD-CCD. Guinier analysis was performed concurrently with isocratic elution of peaks corresponding to the CCD or FHD-CCD, previously verified by SDS-PAGE. The $\mathrm{R}_{\mathrm{g}}$ of $40 \AA$ calculated from the BECN1 CCD crystal structure agrees well with the $\mathrm{R}_{\mathrm{g}}$ of 34-36 Å estimated from the Guinier plot and $\mathrm{P}(\mathrm{r})$ distribution (Figure $\mathrm{S} 3$, Table S1). Consistent with the crystal structure, the $\mathrm{P}(\mathrm{r})$ plot indicates that this is an elongated molecule with a $\mathrm{D}_{\max }$ of $120.6 \AA$ (Figure S3B, Table S1). As expected, the Kratky plot (Figure S3C) indicates that the $\mathrm{CCD}$ is well folded, and theoretical scattering calculated from the CCD crystal structure fit into the molecular envelope calculated from the SAXS data (Table S1, Figure S3D and S3E) agrees with the experimental SAXS curve $\left(\chi^{2}=1.5\right)$. 
SAXS analysis of the FHD-CCD was complicated by the disorder within the FHD. The P(r) plot indicates that the FHD-CCD is a long molecule with a $\mathrm{D}_{\max }$ of $251.9 \AA$ (Figure 6B), consistent with the sum of $\mathrm{D}_{\max }$ expected for a two-fold symmetric model, wherein a FHD flanks the anti-parallel CCD homodimer at each end. The $\mathrm{R}_{\mathrm{g}}$ of the FHD-CCD estimated from the Guinier plot and $\mathrm{P}(\mathrm{r})$ distribution is $70.67 \AA$ (Figure 6 and Table S1), which agrees well with the symmetric structural model. Envelopes for the FHD-CCD were calculated by applying 2-fold symmetry in DAMMIF (57). Each of the envelopes had a long rod similar to the CCD envelope (Figure S3E and Figure S4E), which likely corresponds to the CCD.

Different pseudo-atomic FHD-CCD models were constructed using SASREF (61), by adding residues at the N-termini of each end of the CCD either of three different FHD structures: (i) the FHD crystal structure which did not contain a model for residues for residues 141-156 (ii) a monomer with a disordered $\mathrm{N}$-terminal model extracted from an MD simulation conformer that agreed well with the experimental SAXS data $\left(\chi^{2} \sim 1.0\right)$ and (iii) a monomer with a partially helical $\mathrm{N}$-terminus extracted from an MD simulation conformer that agreed well with the experimental SAXS data $\left(\chi^{2} \sim 1.0\right)$. Relevant parts of the resulting pseudo-atomic model were superimposed upon the individual FHD and CCD envelopes and then the entire model was docked into the FHD-CCD envelope (Figure S4). CRYSOL analysis indicated that the theoretical scattering curves calculated for this FHD-CCD pseudo-atomic model agree well with the FHD-CCD experimental SAXS scattering data (Figure 6D, 6E) with $\chi^{2}$ equal to 1.9 and the agreement with the SAXS data was marginally improved $\left(\chi^{2}=1.7\right)$ when a disordered $\mathrm{N}$-terminus was included in the model, but not if the $\mathrm{N}$-terminal residues were modeled as partly helical $\left(\chi^{2}=2.4\right)$.

Thus our SAXS analysis of the FHD-CCD fragment indicates that there is no interaction between the CCD and FHD that would stabilize ordered structure in the FHD. Furthermore, isothermal titration calorimetry experiments (data not shown) indicate that there is no direct interaction between the FHD and either the BH3D, the N-terminal thirty residues of the $\mathrm{CCD}$, or the entire CCD.

\section{The FHD Likely Undergoes a Binding-Associated Disorder-to-Helix Transition}

The FHD has two invariant residues: Y162 and L169, as well as several highly conserved residues: E139, L144, D146, L148, Q151, E158, and L166 (Figure 1). Of these, residues E158, Y162, L166 and L169 are ordered in the crystal structure. Y162, L166 and L169 map to one face of the FHD helix, while E158 maps to the coil preceding the helix (Figure 2A). Analysis by the program ANCHOR (44) indicates that two regions of the FHD, residues 137-145 and residues 162-169 (Figure 1), may be energetically stabilized in ordered conformations upon binding to an appropriate partner. The BECN1 BH3D, which also contains an Anchor region, has previously been shown to be involved in protein interactions, and to nucleate a disorder-to-helix transition in the BECN1 BH3D upon binding to BCL2 (16). Therefore, we decided to investigate whether the FHD is likely to become completely helical upon binding to a partner.

2,2,2-trifluoroethanol (TFE) is commonly used to mimic the hydrophobic environment experienced by proteins in protein-protein interactions (67-70); therefore, we measured CD spectra for the FHD, CCD, and FHD-CCD in the presence of increasing concentrations of 
TFE to determine if TFE treatment increased helicity. Our CD spectra demonstrate that increasing TFE concentration results in increased helical content in the FHD and FHD-CCD (Figure 7, Table S3), with maximum helicity achieved at 25\% TFE, which was maintained, or slightly decreased, at $40 \%$ TFE. The FHD undergoes a dramatic two-fold increase and the FHD-CCD undergoes a $~ 10 \%$ increase in helicity. In contrast to the FHD-containing BECN1 fragments, helicity of the highly helical CCD barely changes in different concentrations of TFE (Figure 7B and Table S3).

\section{Highly Conserved FHD Residues are Required for Starvation-Induced Up-Regulation of Autophagy}

We decided to investigate the impact of alanine mutagenesis of invariant and highly conserved residues within the FHD (Figure 1); L144, L148, L152, E158, Y162, L166 and L169; on autophagy levels within a cell. As BECN1 is known to be required for autophagosome nucleation, we evaluated cellular autophagy simply by monitoring and comparing levels of puncta labeled with GFP-tagged LC3, an autophagy-specific marker, in cells grown in nutrient-rich and starvation media. These experiments were performed in MCF7 cells that lack one BECN1 allele and express undetectable levels of endogenous BECN1, resulting in very low levels of autophagy $(21,25)$. This enabled us to assess and compare the impact of ectopically-expressed wild-type (WT) or mutant BECN1.

We found that none of the BECN1 FHD mutants impact autophagy levels in nutrient rich media ( $p>0.05$ vs WT), indicating that these conserved FHD residues are not essential for mediating basal autophagy levels. However, these mutations have a much more dramatic impact on starvation-induced up-regulation of autophagy. Mutation of L144, a highlyconserved residue that lies within the first Anchor region, diminishes, but does not completely abrogate, the starvation-induced up-regulation of autophagy ( $\mathrm{p}=0.006$, vs WT). Mutation of another highly conserved residue, E158, which is the first ordered residue in the FHD structure, completely abrogates the starvation-induced up-regulation of autophagy ( $\mathrm{p}=$ $2.5 \mathrm{E}-4$, vs WT) (Figure 8A, 8B). Alanine mutagenesis of the invariant residues within the second Anchor region, Y162, L166, and L169, also completely abrogates the starvationinduced up-regulation of autophagy ( $\mathrm{p}=1.2 \mathrm{E}-4,4.8 \mathrm{E}-4,4 \mathrm{E}-4$ respectively, vs WT) (Figure $8 \mathrm{~A}, 8 \mathrm{~B})$. The only mutations that did not impact cellular autophagy levels were L148A and L152A. Strikingly, both these residues lie outside the two Anchor regions. Thus, residues within the FHD Anchor regions are essential for the starvation-induced up-regulation of autophagy.

\section{DISCUSSION}

Based on the extensive biophysical and structural analyses reported here, we conclude that the BECN1 domain between the $\mathrm{BH} 3 \mathrm{D}$, which constitutes the $\mathrm{C}$-terminal end of the IDR, and the $\mathrm{CCD}$, is partially disordered in the absence of a binding partner. This is consistent with predictions by programs such as IUPRED. Therefore, we have named this BECN1 domain the Flexible-Helical Domain (FHD). SAXS and DEER-EPR analyses indicate that the FHD has an extended conformation while disordered. Furthermore, the FHD does not pack against, or become stabilized in a folded conformation by other BECN1 domains, as 
demonstrated by our data from constructs containing multiple domains along with the FHD. Thus, conformational flexibility in BECN1 extends beyond the IDR, into the FHD.

We further show that the FHD contains two regions, at its N- and C-termini respectively, that are predicted to nucleate folding upon binding, similar to the BH3D that contains a region thought to nucleate folding of that domain (16). If the entire FHD were to become helical, the register of the conserved residues indicates that these conserved residues would map to one face of this helix, further supporting the hypothesis that the FHD folds into a helix upon binding to appropriate partners. This hypothesis is bolstered by the evidence that FHDcontaining BECN1 fragments become more helical in the presence of TFE. Lastly, MD simulations fitted to SAXS data indicate that helicity is maintained at the FHD C-terminus, while the FHD N-terminal disordered region transiently samples helical conformations. A role for the FHD in binding different partners is consistent with the role of BECN1 as a cellular interaction hub that modulates autophagy.

While this manuscript was in preparation, a paper describing the $4.4 \AA$ crystal structure (5DFZ) of full-length yeast VPS30 (BECN1 or ATG6) in a complex comprising VPS34 (PI3KC3), VPS15 (p150) and VPS38 (UVRAG) was published (71). Consistent with our results, there is no intra-molecular interaction between the VPS30 region (labeled CC1 in this structure) that is equivalent to the FHD, and any other BECN1 domain. In this complex the VPS30 CC1 forms an eight-turn helix and, as part of a coiled-coil heterodimer, is packed against a helix of VPS38, consistent with our results suggesting that this region undergoes a binding-associated disorder-to-helix transition. Curiously however, conserved FHD residues do not map to the interface with VPS38, but are completely solvent-exposed. This would allow the FHD to be responsible for nucleating other interactions that modulate the activity of the PI3KC3 complex. Indeed, this BECN1 region has been shown to be required for interaction with another important autophagy protein AMBRA1 $(72,73)$. It would be interesting to determine if AMBRA1 can bind to BECN1 in the absence of binding to UVRAG (or ATG14), or whether the BECN1 FHD would need to be stabilized in a helical state prior to AMBRA1 binding.

Strikingly, we show that single point mutations of highly conserved FHD residues completely abrogate the starvation-induced up-regulation of autophagy, but have no effect on the basal level of autophagy. This implies that the FHD is responsible for transmitting signals for the up-regulation of autophagy in response to nutrient deprivation. To our knowledge, this is the first BECN1 region to be directly implicated in up-regulating autophagy in response to starvation, rather than impacting general autophagy levels. Both AMBRA1 and UVRAG have previously been shown to also be important for the upregulation of autophagy $(8,72,74)$. This may suggest that mutation of conserved BECN1 FHD residues abolishes the interaction with either AMBRA1 or UVRAG, or perhaps yet some other uncharacterized interaction, thereby abrogating the starvation-induced upregulation of autophagy.

In the crystal structure reported here, the FHD forms a trimer that is stabilized by the partial packing of conserved hydrophobic residues. MD simulations fitted to SAXS data suggest that this trimer likely exists in solution. However, the FHD is unlikely to trimerize in this 
manner in the context of the PI3KC3 complex, as there would be extensive stearic clashes between three PI3KC3 complexes arranged based on superposition of the VPS30 CC1 on the FHD. Therefore, it is likely that this FHD trimer represents alternate FHD structure and oligomerization, in BECN1 states accessed while performing other biological functions. Notably, regions N-terminal to the CCD have been implicated in homo-oligomerization in other studies $(71,75)$, and it has been shown that the levels of homo-oligomerized BECN1 molecules in cells are not affected by induction of autophagy and association with the other core components of the PI3KC3 complex (75). Lastly, it is tempting to speculate that the sulfate-binding site created by the formation of this trimer may represent a site of interaction with a phosphorylated partner.

BECN1 has important roles in cellular pathways that do not involve the other core proteins of the PI3KC3 complex. For instance, BECN1 has been shown to localize to the nucleus (21). It is unclear whether BECN1 interacts with other proteins in the nucleus, but it appears to impact nuclear export of other autophagy proteins such as LC3 that are not part of the PI3KC3 complex (22). BECN1 proteolytic fragments have also been implicated in apoptosis $(27,28)$. The BECN1 FHD structure discussed here may have a role in either of these biological functions. Disorder-to-order transitions in different BECN1 regions have been shown to be essential for regulation of BECN1 function in autophagy $(16,76)$. However, the mechanism by which BECN1 mediates its other biological functions, as well as the mechanism of regulation of these diverse BECN1 functions, is not understood. Thus, it is essential that we continue to explore and understand the structure and conformational flexibility of BECN1 in the absence of interactions with other proteins, as well as in the context of diverse interactions, to fully understand the mechanism by which BECN1 performs this diverse array of biological functions.

\section{Supplementary Material}

Refer to Web version on PubMed Central for supplementary material.

\section{Acknowledgments}

We thank Dr. Wayne Hubbell at University of California, Los Angeles for access to EPR instrumentation. We also thank the 2012 CCP4/APS crystallographic school held at APS (http://www.ccp4.ac.uk/schools/APS-school/ index.php), especially Kai Diederiches (Universität Konstanz), at which single crystal X-ray diffraction data collection and structure determination were carried out. The authors also acknowledge the NDSU Advanced Imaging and Microscopy Core Laboratory for access to microscopy and imaging equipment conducted in this study. This work was supported by NIH grants RO3 NS090939 (S.S.) and R15 GM113227 (C.C.); a National Science Foundation grant MCB-1413525 (S.S.); a North Dakota EPSCoR doctoral dissertation award for Y.M. (S.S.); and a Laboratory Director's Research and Development SEED proposal 7278 (A.R.). Usage of the Core Biology Facility was funded by NIH grant P30 GM103332-01. Work performed at Bio-CAT was supported by NIH NIGMS 9P41 GM103622. GM/CA@APS has been funded in whole or in part with Federal funds from the NCI (ACB-12002) and the National Institute of General Medical Sciences (AGM-12006). This research used resources of the APS, a U.S. DOE Office of Science User Facility operated for the DOE Office of Science by Argonne National Laboratory under Contract No. DE-AC02-06CH11357. ORNL is operated by UT-Battelle, LLC, for the U.S. Department of Energy under contract DE-AC05-00OR22725. This research used resources of the Oak Ridge Leadership Computing Facility at ORNL. 


\section{ABBREVIATIONS}

\begin{tabular}{ll} 
BECN1 & BCL-2 interacting protein or Beclin 1 \\
BH3 & BCL2 homology 3 \\
FHD & flexible helical domain \\
CCD & coiled-coil domain \\
BARAD & -a-repeat autophagy-specific domain \\
SAD & single-wavelength anomalous dispersion \\
IPTG & isopropyl thio- $\beta$-D-galactoside \\
TEV & tobacco etch virus \\
SEC & size exclusion chromatography \\
CD & circular dichroism spectroscopy \\
DEER-EPR & Double-Electron-Electron Resonance-Electron Paramagnetic Resonance \\
SAXS & small-angle X-ray scattering \\
MD & Molecular dynamic \\
IPTG & isopropyl thio- $\beta$-D-galactoside \\
SDS-PAGE & sodium dodecyl sulfate polyacrylamide gel electrophoresis \\
MBP & maltose binding protein \\
TFE & $2,2,2$-trifluoroethanol \\
RMSD & root-mean-square deviation \\
R & radius of gyration \\
P(r) & pairwise distribution function \\
D & maximum particle distribution \\
WT & wild-type \\
\hline
\end{tabular}

\section{REFERENCES}

1. Levine B, Klionsky DJ. Development by self-digestion: Molecular mechanisms and biological functions of autophagy. Dev. Cell. 2004; 6:463-477. [PubMed: 15068787]

2. Xie Z, Klionsky DJ. Autophagosome formation: core machinery and adaptations. Nat Cell Biol. 2007; 9

3. Mizushima N, Yoshimori T, Ohsumi Y. The role of Atg proteins in autophagosome formation. Annu. Rev. Cell Dev. Biol. 2011; 27:107-132. [PubMed: 21801009]

4. Shintani T, Klionsky DJ. Autophagy in health and disease: A double-edged sword. Science. 2004; 306:990-995. [PubMed: 15528435]

5. Levine B, Kroemer G. Autophagy in the pathogenesis of disease. Cell. 2008; 132:27-42. [PubMed: 18191218]

6. Kroemer G, Levine B. Autophagic cell death: the story of a misnomer. Nat Rev Mol Cell Biol. 2008; 9:1004-1010. [PubMed: 18971948] 
7. Mizushima N, Levine B, Cuervo A, Klionsky D. Autophagy fights disease through cellular selfdigestion. Nature. 2008; 451:1069-1075. [PubMed: 18305538]

8. Liang C, Feng P, Ku B, Dotan I, Canaani D, Oh BH, Jung JU. Autophagic and tumour suppressor activity of a novel Beclin1-binding protein UVRAG. Nat Cell Biol. 2006; 8:688-699. [PubMed: 16799551]

9. Itakura E, Kishi C, Inoue K, Mizushima N. Beclin 1 forms two distinct phosphatidylinositol 3kinase complexes with mammalian Atg14 and UVRAG. Molecular biology of the Cell. 2008; 19:5360-5372. [PubMed: 18843052]

10. Zhong Y, Wang QJ, Li XT, Yan Y, Backer JM, Chait BT, Heintz N, Yue ZY. Distinct regulation of autophagic activity by Atg14L and Rubicon associated with Beclin 1-phosphatidylinositol-3kinase complex. Nature Cell Biology. 2009; 11:468-476. [PubMed: 19270693]

11. Matsunaga K, Saitoh T, Tabata K, Omori H, Satoh T, Kurotori N, Maejima I, Shirahama-Noda K, Ichimura T, Isobe T, Akira S, Noda T, Yoshimori T. Two Beclin 1-binding proteins, Atg14L and Rubicon, reciprocally regulate autophagy at different stages. Nature Cell Biology. 2009; 11:385396. [PubMed: 19270696]

12. Itakura E, Mizushima N. Atg14 and UVRAG: mutually exclusive subunits of mammalian Beclin 1PI3K complexes. Autophagy. 2009; 5:534-536. [PubMed: 19223761]

13. Behrends C, Sowa ME, Gygi SP, Harper JW. Network organization of the human autophagy system. Nature. 2010; 466:68-76. [PubMed: 20562859]

14. He C, Levine B. The Beclin 1 interactome. Current Opinion in Cell Biology. 2010; 22:140-149. [PubMed: 20097051]

15. Kang R, Zeh H, Lotze M, Tang D. The Beclin 1 network regulates autophagy and apoptosis. Cell Death and Differentiation. 2011; 18:571-580. [PubMed: 21311563]

16. Mei Y, Su M, Soni G, Salem S, Colbert C, Sinha S. Intrinsically disordered regions in autophagy proteins. PROTEINS: Structure, Function and Bioinformatics. 2014; 82:565-578.

17. Levine B, Sinha S, Kroemer G. Bcl-2 family members: dual regulators of apoptosis and autophagy. Autophagy. 2008; 4:600-606. [PubMed: 18497563]

18. Orvedahl, A.; Levine, B. Autophagy in Mammalian Antiviral Immunity. In: Levine, B.; Yoshimori, T.; Deretic, V., editors. Autophagy in Infection and Immunity. Berlin Heidelberg, Heidelberg: Springer-Verlag; 2009. p. 251-266.

19. Levine B, Deretic V. Unveiling the roles of autophagy in innate and adaptive immunity. Nat. Rev. Immunol. 2007; 7:767-777. [PubMed: 17767194]

20. Gannagé M, Rämer PC, Munz C. Targeting Beclin 1 for viral subversion of macroautophagy. Autophagy. 2010; 6:166-167. [PubMed: 20009549]

21. Liang XH, Yu J, Brown K, Levine B. Beclin 1 contains a leucine-rich nuclear export signal that is required for its autophagy and tumor suppressor function. Cancer Res. 2001; 61:3443-3449. [PubMed: 11309306]

22. Ma X, Liu H, Murphy JT, Foyil SR, Godar RJ, Abuirqeba H, Weinheimer CJ, Barger PM, Diwan A. Regulation of the transcription factor EB-PGC1alpha axis by beclin-1 controls mitochondrial quality and cardiomyocyte death under stress. Mol Cell Biol. 2015; 35:956-976. [PubMed: 25561470]

23. Koukourakis MI, Kalamida D, Giatromanolaki A, Zois CE, Sivridis E, Pouliliou S, Mitrakas A, Gatter KC, Harris AL. Autophagosome Proteins LC3A, LC3B and LC3C Have Distinct Subcellular Distribution Kinetics and Expression in Cancer Cell Lines. PLoS ONE. 2015; 10:e0137675. [PubMed: 26378792]

24. Su M, Mei Y, Sinha S. Role of the Crosstalk between Autophagy and Apoptosis in Cancer. Journal of Oncology. 2013; 2013:102735. [PubMed: 23840208]

25. Pattingre S, Tassa A, Qu X, Garuti R, Liang XH, Mizushima N, Packer M, Schneider MD, Levine B. Bcl-2 antiapoptotic proteins inhibit Beclin 1-dependent autophagy. Cell. 2005; 122:927-939. [PubMed: 16179260]

26. Sinha S, Levine B. The autophagy effector Beclin 1: A novel BH3-only protein. Oncogene. 2009; 27:S137-S148. [PubMed: 19641499] 
27. Cho D-H, Jo YK, Hwang JJ, Lee YM, Roh SA, Kim JC. Caspase-mediated cleavage of ATG6/ Beclin-1 links apoptosis to autophagy in HeLa cells. Cancer Letters. 2009; 274:95-100. [PubMed: 18842334]

28. Wirawan E, Vande Walle L, Kersse K, Cornelis S, Claerhout S, Vanoverberghe I, Roelandt R, De Rycke R, Verspurten J, Declercq W, Agostinis P, Vanden Berghe T, Lippens S, Vandenabeele P. Caspase-mediated cleavage of Beclin-1 inactivates Beclin-1-induced autophagy and enhances apoptosis by promoting the release of proapoptotic factors from mitochondria. Cell Death Dis. 2010; 1

29. Oberstein A, Jeffrey PD, Shi Y. Crystal structure of the Bcl-XL-Beclin 1 peptide complex: Beclin 1 is a novel BH3 only protein. The Journal of Biological Chemistry. 2007; 282:13123-13132. [PubMed: 17337444]

30. Feng W, Huang $\mathrm{S}$, Wu H, Zhang M. Molecular basis of Bcl- $\mathrm{X}_{\mathrm{L}}$ 's target recognition versatility revealed by the structure of Bcl- $\mathrm{X}_{\mathrm{L}}$ in complex with the $\mathrm{BH} 3$ domain of Beclin-1. J. Mol. Biol. 2007; 372:223-235. [PubMed: 17659302]

31. Sinha S, Colbert CL, Becker N, Wei Y, Levine B. Molecular basis of the regulation of Beclin 1dependent autophagy by the $\gamma$-herpesvirus 68 Bcl-2 homolog M11. Autophagy. 2008; 4:989-997. [PubMed: 18797192]

32. Ku B, Woo J-S, Liang C, Lee K-H, Hong H-S, Xiaofei E, Kim K-S, Jung JU, Oh B-H. Structural and biochemical bases for the inhibition of autophagy and apoptosis by viral Bcl-2 of murine $\gamma$ Herpesvirus 68. PLoS Pathogens. 2008; 4:e25. [PubMed: 18248095]

33. Li X, He L, Che KH, Funderburk SF, Pan L, Pan N, Zhang M, Yue Z, Zhao Y. Imperfect interface of Beclin1 coiled-coil domain regulates homodimer and heterodimer formation with Atg14L and UVRAG. Nature communicaitons. 2012; 3:662.

34. Huang W, Choi W, Hu W, Mi N, Guo Q, Ma M, Liu M, Tian Y, Lu P, Wang F-L, Deng H, Liu L, Gao N, Yu L, Shi Y. Crystal structure and biochemical analyses reveal Beclin 1 as a novel membrane binding protein. Cell Research. 2012; 22:473-489. [PubMed: 22310240]

35. Noda NN, Kobayashi T, Adachi W, Fujioka Y, Ohsumi Y, Inagaki F. Structure of the novel Cterminal domain of vacuolar protein sorting 30/autophagy-related protein 6 and its specific role in autophagy. The Journal of biological chemistry. 2012; 287:16256-16266. [PubMed: 22437838]

36. Furuya N, Yu F, Byfield M, Pattingre S, Levine B. The evolutionarily conserved domain of Beclin 1 is required for Vps34 binding, autophagy and tumor suppressor function. Autophagy. 2005; 1:46-52. [PubMed: 16874027]

37. Maiuri M, Criollo A, Tasdemir E, Vicencio J, Tajeddine N, Hickman J, Geneste O, Kroemer G. $\mathrm{BH} 3$-only proteins and $\mathrm{BH} 3$ mimetics induce autophagy by competitively disrupting the interaction between Beclin 1 and Bcl-2/Bcl-X $\mathrm{L}_{\mathrm{L}}$. Autophagy. 2007; 3:374-476. [PubMed: 17438366]

38. Su M, Mei Y, Sanishvili R, Levine B, Colbert CL, Sinha S. Targeting $\gamma$-herpesvirus 68 Bcl-2mediated down-regulation of autophagy. Journal of Biological Chemistry. 2014; 289:8029-8050. [PubMed: 24443581]

39. Baskaran S, Carlson L-A, Stjepanovic G, Young LN, Kim DJ, Grob P, Stanley RE, Nogales E, Hurley JH. Architecture and dynamics of the autophagic phosphatidylinositol 3-kinase complex. eLife. 2014; 3

40. Liang XH, Jackson S, Seaman M, Brown K, Kempkes B, Hibshoosh H, Levine B. Induction of autophagy and inhibition of tumorigenesis by Beclin 1. Nature. 1999; 402:672-676. [PubMed: 10604474]

41. Sheffield P, Garrard S, Derewenda Z. Overcoming expression and purification problems of RhoGDI using a family of "Parallel" expression vectors. Prot. Express. and Purif. 1999; 15:34-39.

42. Meszaros B, Simon I, Dosztanyi Z. Prediction of protein binding regions in disordered proteins. PLoS Computational Biology. 2009; 5:e1000376. [PubMed: 19412530]

43. Dosztányi Z, Csizmok V, Tompa P, Simon In. IUPred: web server for the prediction of intrinsically unstructured regions of proteins based on estimated energy content. Bioinformatics. 2005; 21:3433-3434. [PubMed: 15955779]

44. Dosztányi Z, Mészáros B, Simon I. ANCHOR: web server for predicting protein binding regions in disordered proteins. Bioinformatics. 2009; 25:2745-2746. [PubMed: 19717576] 
45. Kabsch W. Integration, scaling, space-group assignment and post-refinement. Acta Crystallogr D. 2010; 66:133-144. [PubMed: 20124693]

46. Sheldrick GM. Experimental phasing with SHELXC/D/E: combining chain tracing with density modification. Acta Crystallogr D. 2010; 66:479-485. [PubMed: 20383001]

47. Emsley P, Lohkamp B, Scott WG, Cowtand K. Features and development of Coot. Acta crystallographica. Section D, Biological crystallography. 2010; 66:486-501. [PubMed: 20383002]

48. Adams PD, Afonine PV, Bunkoczi G, Chen VB, Davis IW, Echols N, Headd JJ, Hung LW, Kapral GJ, Grosse-Kunstleve RW, McCoy AJ, Moriarty NW, Oeffner R, Read RJ, Richardson DC, Richardson JS, Terwilliger TC, Zwart PH. PHENIX: a comprehensive Python-based system for macromolecular structure solution. Acta crystallographica. Section D, Biological crystallography. 2010; 66:213-221. [PubMed: 20124702]

49. Sreerama N, Venyaminov SY, Woody RW. Analysis of protein circular dichroism spectra based on the tertiary structure classification. Analytical biochemistry. 2001; 299:271-274. [PubMed: 11730356]

50. Hubbell WL, Lopez CJ, Altenbach C, Yang Z. Technological advances in site-directed spin labeling of proteins. Curr Opin Struct Biol. 2013; 23:725-733. [PubMed: 23850140]

51. Lopez CJ, Yang Z, Altenbach C, Hubbell WL. Conformational selection and adaptation to ligand binding in T4 lysozyme cavity mutants. Proc Natl Acad Sci U S A. 2013; 110:E4306-E4315. [PubMed: 24167295]

52. Jeschke G. DEER Distance Measurements on Proteins. Annu Rev Phys Chem. 2012; 63:419-446. [PubMed: 22404592]

53. Ilavsky J, Jemian PR. Irena: tool suite for modeling and analysis of small-angle scattering. Journal of Applied Crystallography. 2009; 42:347-353.

54. Petoukhov MV, Franke D, Shkumatov AV, Tria G, Kikhney AG, Gajda M, Gorba C, Mertens HDT, Konarev PV, Svergun DI. New developments in the ATSAS program package for small-angle scattering data analysis. Journal of Applied Crystallography. 2012; 45:342-350. [PubMed: 25484842]

55. Konarev PV, Volkov VV, Sokolova AV, Koch MHJ, Svergun DI. PRIMUS: a Windows PC-based system for small-angle scattering data analysis. Journal of Applied Crystallography. 2003; 36:1277-1282.

56. Svergun DI. Determination of the regularization parameter in indirect-transform methods using perceptual criteria. Journal of Applied Crystallography. 1992; 25:495-503.

57. Franke D, Svergun DI. DAMMIF, a program for rapid ab-initio shape determination in small-angle scattering. Journal of Applied Crystallography. 2009; 42:342-346.

58. Volkov VV, Svergun DI. Uniqueness of ab initio shape determination in small-angle scattering. Journal of Applied Crystallography. 2003; 36:860-864.

59. Svergun D, Barberato C, Koch MHJ. CRYSOL - A program to evaluate x-ray solution scattering of biological macromolecules from atomic coordinates. Journal of Applied Crystallography. 1995; 28:768-773.

60. Kozin MB, Svergun DI. Automated matching of high- and low-resolution structural models. Journal of Applied Crystallography. 2001; 34:33-41.

61. Petoukhov MV, Svergun DI. Global rigid body modeling of macromolecular complexes against small-angle scattering data. Biophys J. 2005; 89:1237-1250. [PubMed: 15923225]

62. Ramanathan A, Agarwal PK. Computational identification of slow conformational fluctuations in proteins. J. Phys. Chem. B. 2009; 113:16669-16680. [PubMed: 19908896]

63. Kabeya Y, Mizushima N, Ueno T, Yamamoto A, Kirisako T, Noda T, Kominami E, Ohsumi Y, Yoshimori T. LC3, a mammalian homologue of yeast Apg8p, is localized in autophagosome membranes after processing. The EMBO Journal. 2000; 19:5720-5728. [PubMed: 11060023]

64. Sreerama N, Woody RW. A self-consistent method for the analysis of protein secondary structure from circular dichroism. Anal Biochem. 1993; 209:32-44. [PubMed: 8465960]

65. Sreerama N, Woody RW. Estimation of protein secondary structure from circular dichroism spectra: comparison of CONTIN, SELCON, and CDSSTR methods with an expanded reference set. Anal Biochem. 2000; 287:252-260. [PubMed: 11112271] 
66. Chen, P-c; Hub, JS. Validating solution ensembles from molecular dynamics simulations by wideangle X-ray scattering data. Biophysical Journal. 2014; 107:435-447. [PubMed: 25028885]

67. Buck M, Radford SE, Dobson CM. A partially folded state of hen egg white lysozyme in trifluoroethanol: structural characterization and implications for protein folding. Biochemistry. 1993; 32:669-678. [PubMed: 8422374]

68. Buck M. Trifluoroethanol and colleagues: cosolvents come of age. Recent studies with peptides and proteins. Quarterly reviews of biophysics. 1998; 31:297-355. [PubMed: 10384688]

69. Roccatano D, Colombo G, Fioroni M, Mark AE. Mechanism by which 2,2,2-trifluoroethanol/water mixtures stabilize secondary-structure formation in peptides: A molecular dynamics study. Proceedings of the National Academy of Sciences of the United States of America. 2002; 99:12179-12184. [PubMed: 12196631]

70. Hua QX, Jia WH, Bullock BP, Habener JF, Weiss MA. Transcriptional activator-coactivator recognition: Nascent folding of a kinase-inducible transactivation domain predicts its structure on coactivator binding. Biochemistry. 1998; 37:5858-5866. [PubMed: 9558319]

71. Rostislavleva K, Soler N, Ohashi Y, Zhang L, Pardon E, Burke JE, Masson GR, Johnson C, Steyaert J, Ktistakis NT, Williams RL. Structure and flexibility of the endosomal Vps34 complex reveals the basis of its function on membranes. Science. 2015; 350:aac7365. [PubMed: 26450213]

72. Fimia G, Stoykova A, Romagnoli A, Giunta L, Bartolomeo S, Nardacci R, Corazzari M, Fuoco C, Ucar A, Schwartz P, Gruss P, Piacentini M, Chowdhury K, Cecconi F. Ambra1 regulates autophagy and development of the nervous system. Nature. 2007; 447:1121-1125. [PubMed: 17589504]

73. Strappazzon F, Vietri-Rudan M, Campello S, Nazio F, Florenzano F, Fimia GM, Piacentini M, Levine B, Cecconi F. Mitochondrial BCL-2 inhibits AMBRA1-induced autophagy. The EMBO journal. 2011; 30:1195-1208. [PubMed: 21358617]

74. Gu W, Wan D, Qian Q, Yi B, He Z, Gu Y, Wang L, He S. Ambra1 is an essential regulator of autophagy and apoptosis in SW620 cells: pro-survival role of Ambra1. PloS One. 2014; 9:e90151. [PubMed: 24587252]

75. Adi-Harel S, Erlich S, Schmukler E, Cohen-Kedar S, Segev O, Mizrachy L, Hirsch JA, PinkasKramarski R. Beclin 1 self-association is independent of autophagy induction by amino acid deprivation and rapamycin treatment. J Cell Biochem. 2010; 110:1262-1271. [PubMed: 20564222]

76. Wei Y, An Z, Zou Z, Sumpter R, Su M, Zang X, Sinha S, Gaestel M, Levine B. The stressresponsive kinases MAPKAPK2/MAPKAPK3 activate starvation-induced autophagy through Beclin 1 phosphorylation. LID -10.7554/eLife.05289 [doi]. eLife. 2015; 4 
Anchor:

137-145

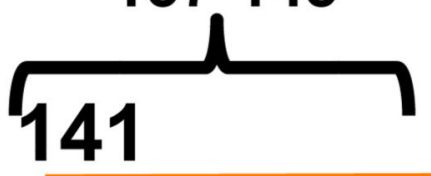

Anchor:

162-169

157

Human

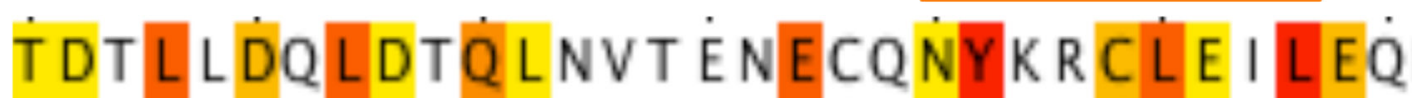

Mouse TDTLLDQLDTQLNVTENECQNYKRCLE I LEQ

Chicken TDT LLDQLDTQLNITENECQNYKRCLE I LEK

Frog

Fly

Worm

Plant

Yeast

TDT

ADSMLEIMDRELR I AEDEWDVYKAY LDELEQ

SDALRNEMDAQVATLDDE I KTYQTY I NY LKE

MRVLSDKLEKEVEDVTRDVEAYEACVQRLEG

CN I L I NR LKSEYDDA I KERDTYAQF LSKLES

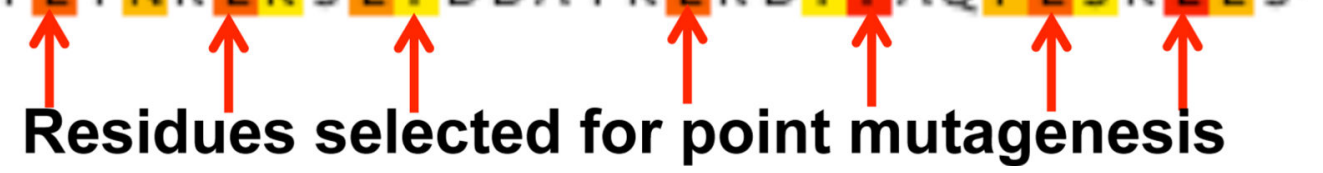

Figure 1.

Sequence alignment of the BECN1 FHD from eight diverse eukaryotes. Residue numbers correspond to the human FHD. Yellow, orange and red backgrounds represent increasing sequence conservation with red corresponding to invariant residues. Secondary structure elements reported in this paper are displayed above the alignment with the orange line representing the disordered region and the orange cylinder representing the helical region. Residues predicted by the program ANCHOR (44) to nucleate folding upon binding are indicated. Arrows indicate residues that were mutated to Ala for cellular assays. 
A

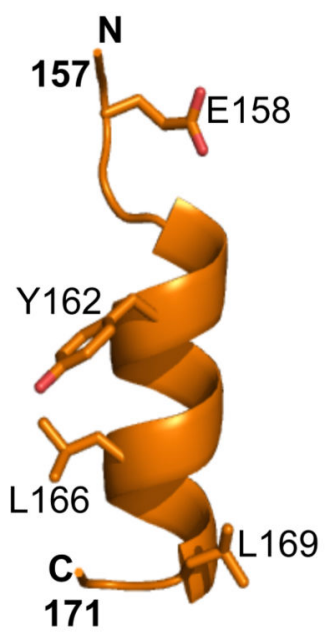

B

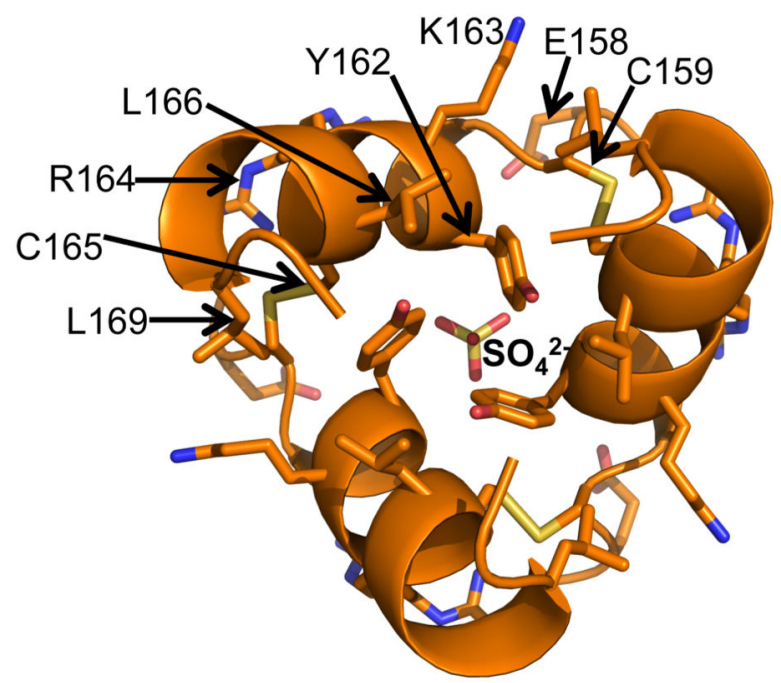

C

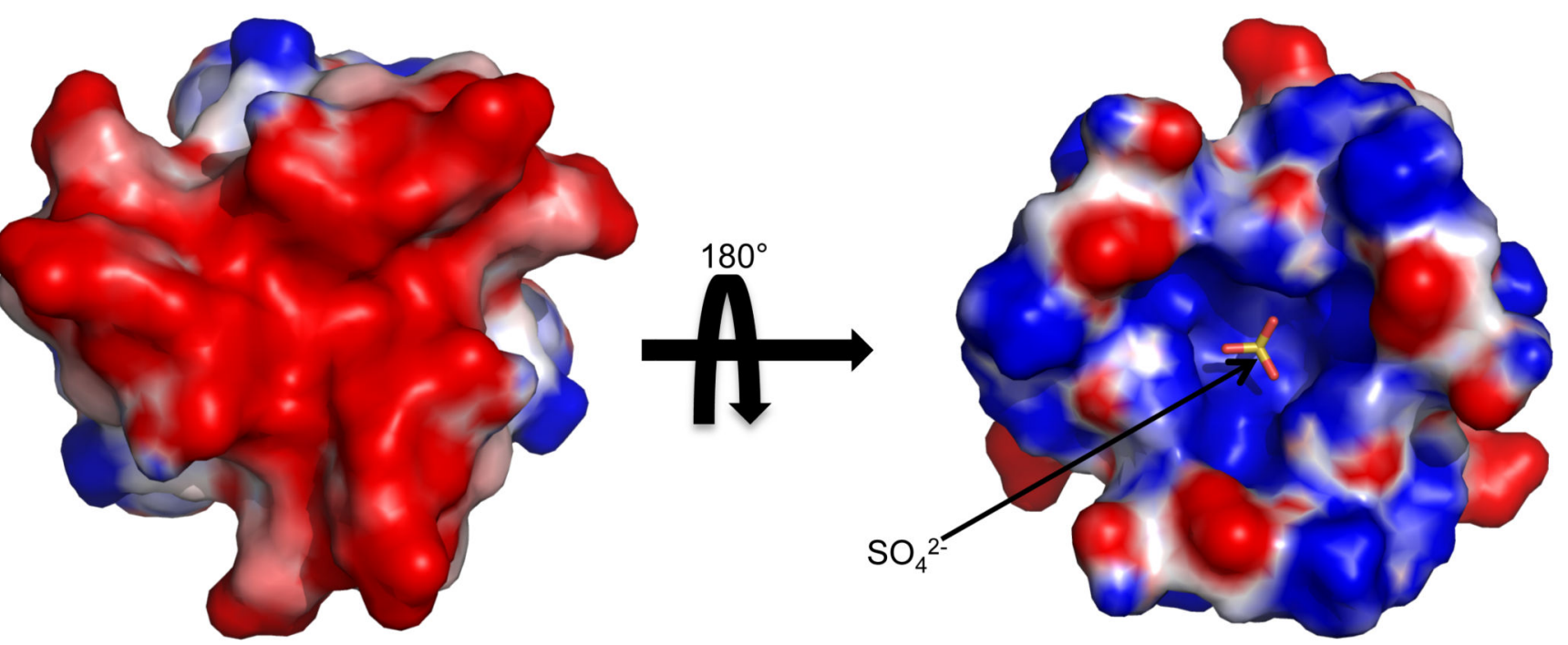

Figure 2.

The FHD crystal structure. (A) The FHD monomer. The FHD backbone is displayed in orange ribbon, while highly conserved residues are labeled and rendered in stick, with atoms color-coded by type: C, orange; O, red; N, blue and S, yellow. This and all other molecular figures were made using PyMol. (B) The FHD trimer showing three molecules arranged around the crystallographic 3-fold are rendered as in (A). Highly conserved or structurally important residues from one monomer are labeled. (C) Electrostatic potential surface of the FHD trimer calculated using APBS. The figure on the left displays the face bearing the Ctermini of the FHD monomers. The figure on the right displays the face bearing the Ntermini of the FHD monomers. This face has a pocket that binds a sulfate molecule, rendered in stick and color-coded by atom type as in (A). 

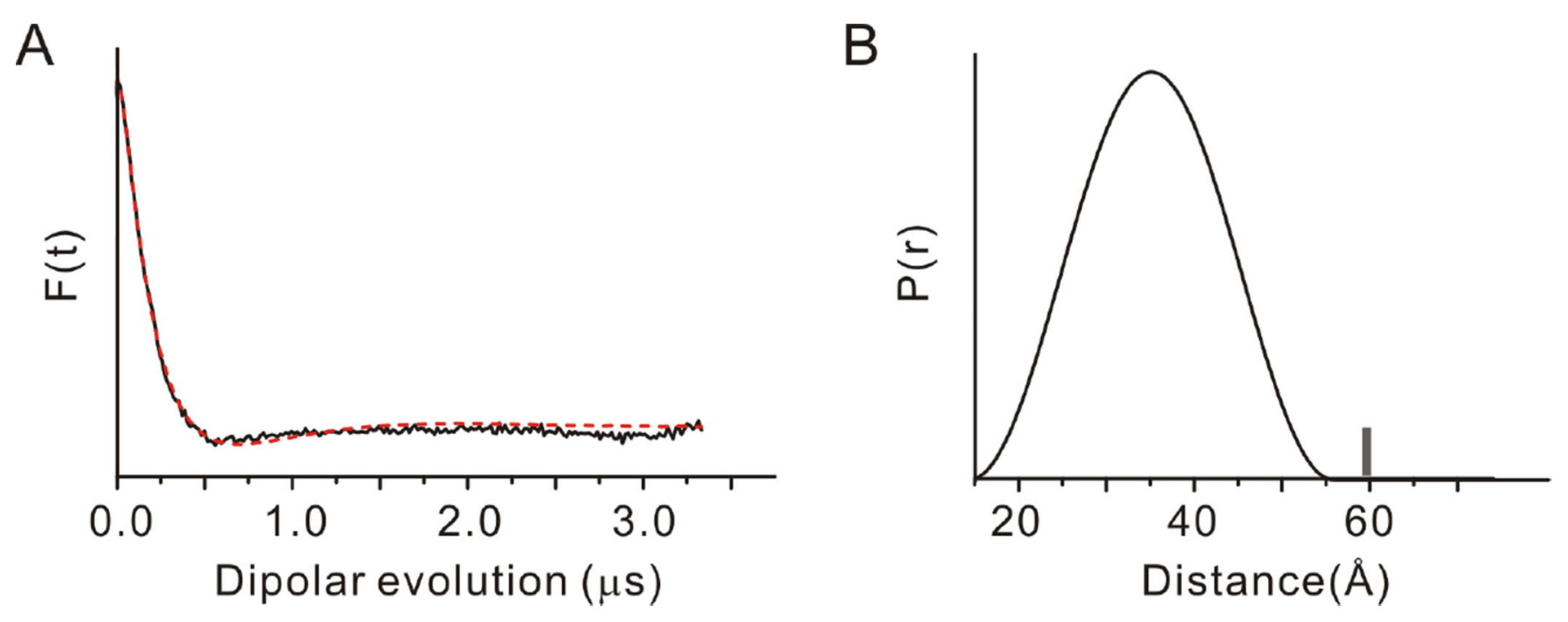

Figure 3.

DEER-EPR data and data analysis on the spin labeled FHD. (A) The DEER signal intensity, $\mathrm{F}(\mathrm{t})$, as a function of time domain dipolar evolution (black curve). The data is analyzed by assuming that the distance distribution between the spin labels is a Gaussian function with the best fit shown by the red dotted curve. (B) The distance distribution function, $\mathrm{P}(\mathrm{r})$, derived from the analysis of the time domain signal in (A). The grey vertical bars indicate the approximate upper limit of reliable distances determined from DEER. 
A

B C
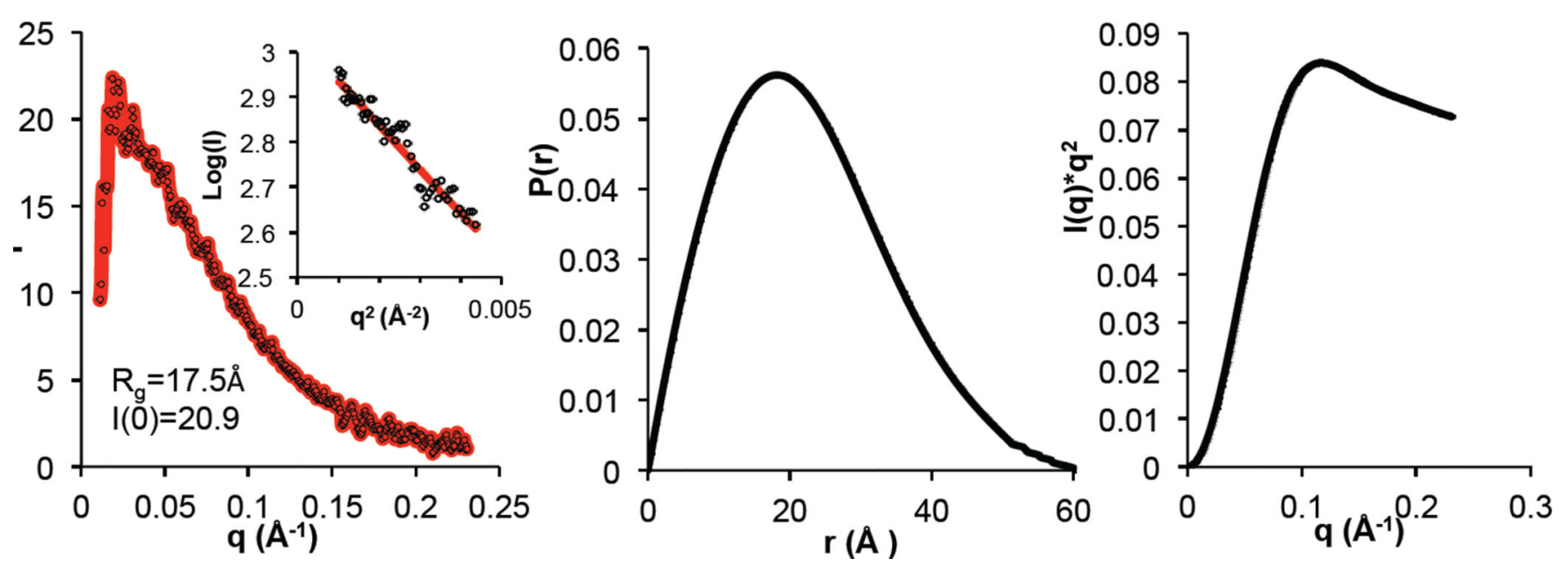

Figure 4.

SAXS analysis of the BECN1 FHD. (A) Guinier Plot. (B) P(r) pairwise distribution. (C) Kratky plot. 


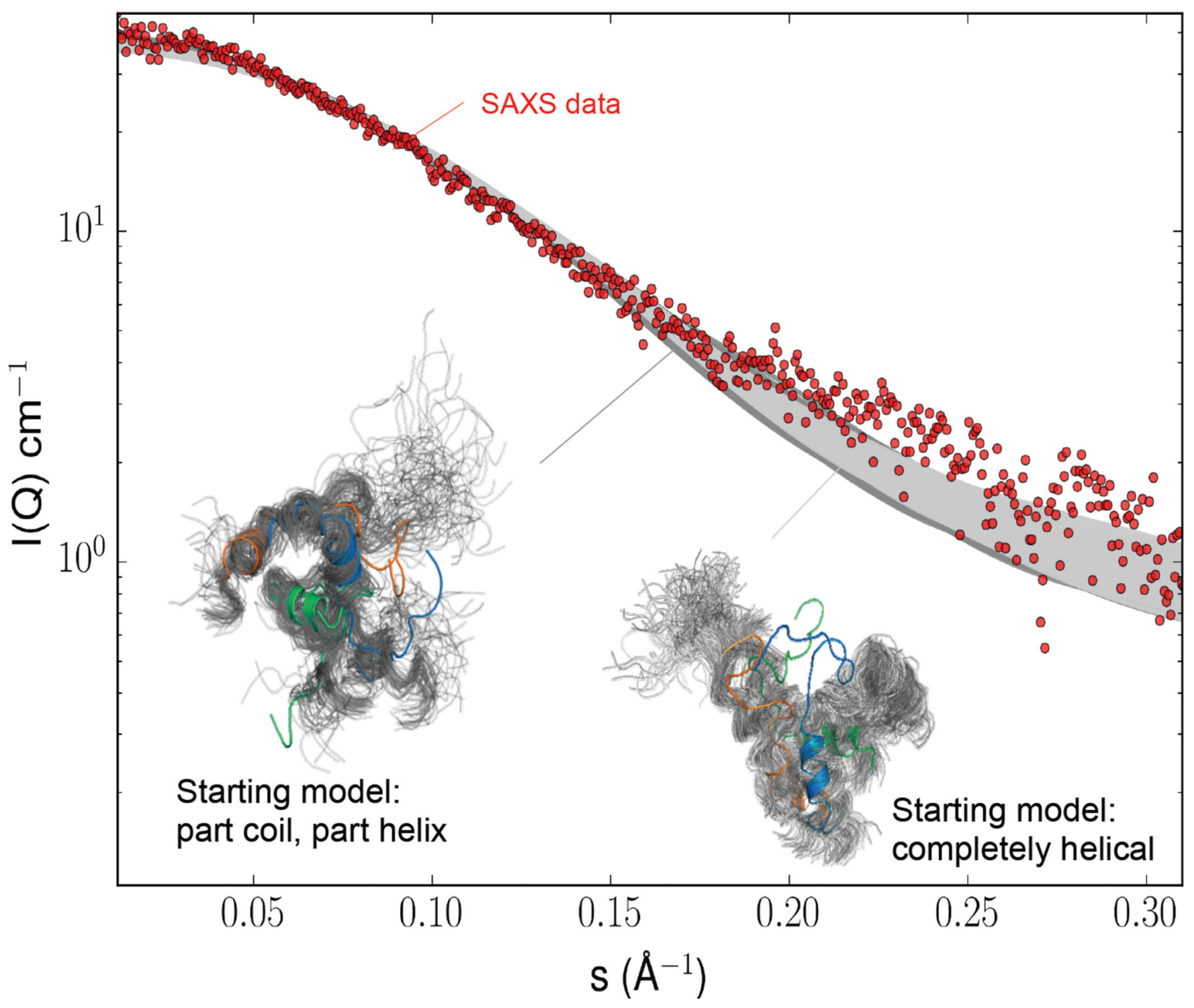

Figure 5.

MD conformer ensembles of the FHD fit to experimental SAXS data. The fits to the experimental SAXS data (red dots) are shown in dark grey and light grey for the MD simulations initiated from the part coil and part helical FHD trimer (left ensemble) and for the simulations initiated from the fully helical FHD trimer (right ensemble) respectively. An ensemble representation of conformers that show a good fit $\left(0.5 \leq \chi^{2} \leq 1.5\right)$ to the experimental SAXS data is shown for each set of simulations. 
A

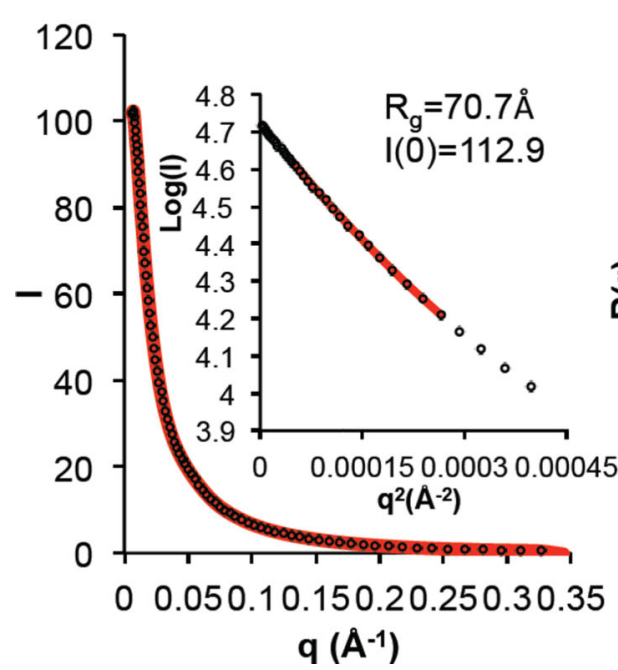

B

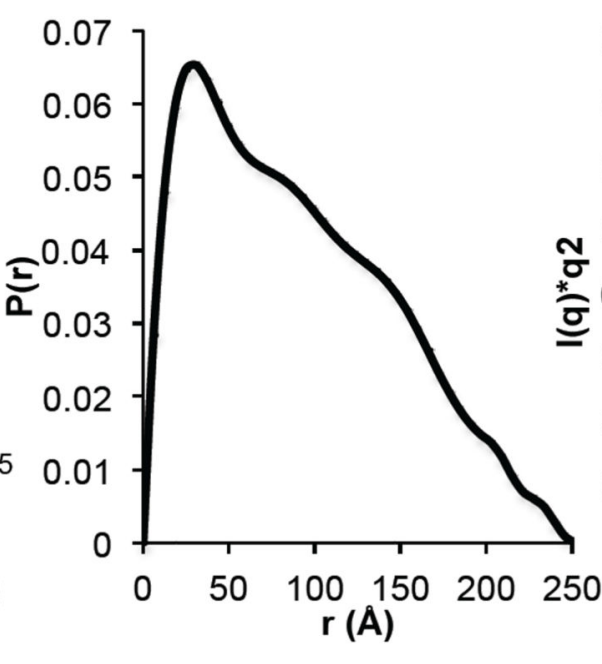

C

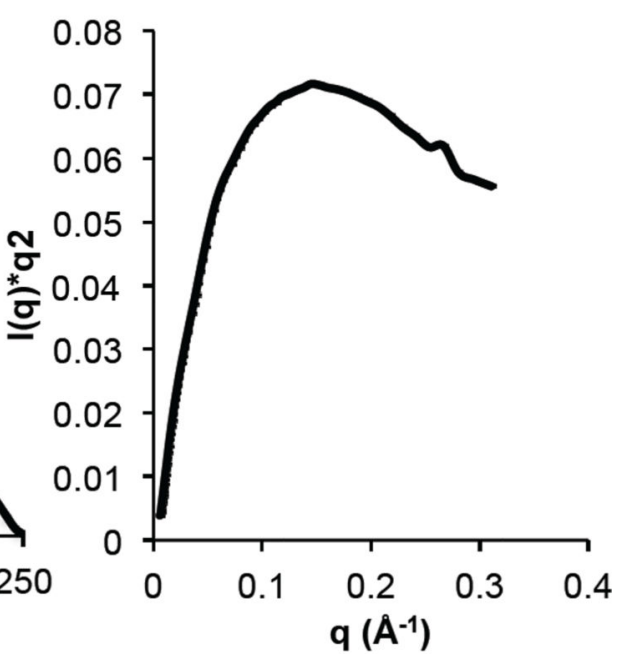

Figure 6.

SAXS analysis of BECN1 FHD-CCD. (A) Guinier Plot. (B) P(r) pairwise distribution (C) Kratky plot. 

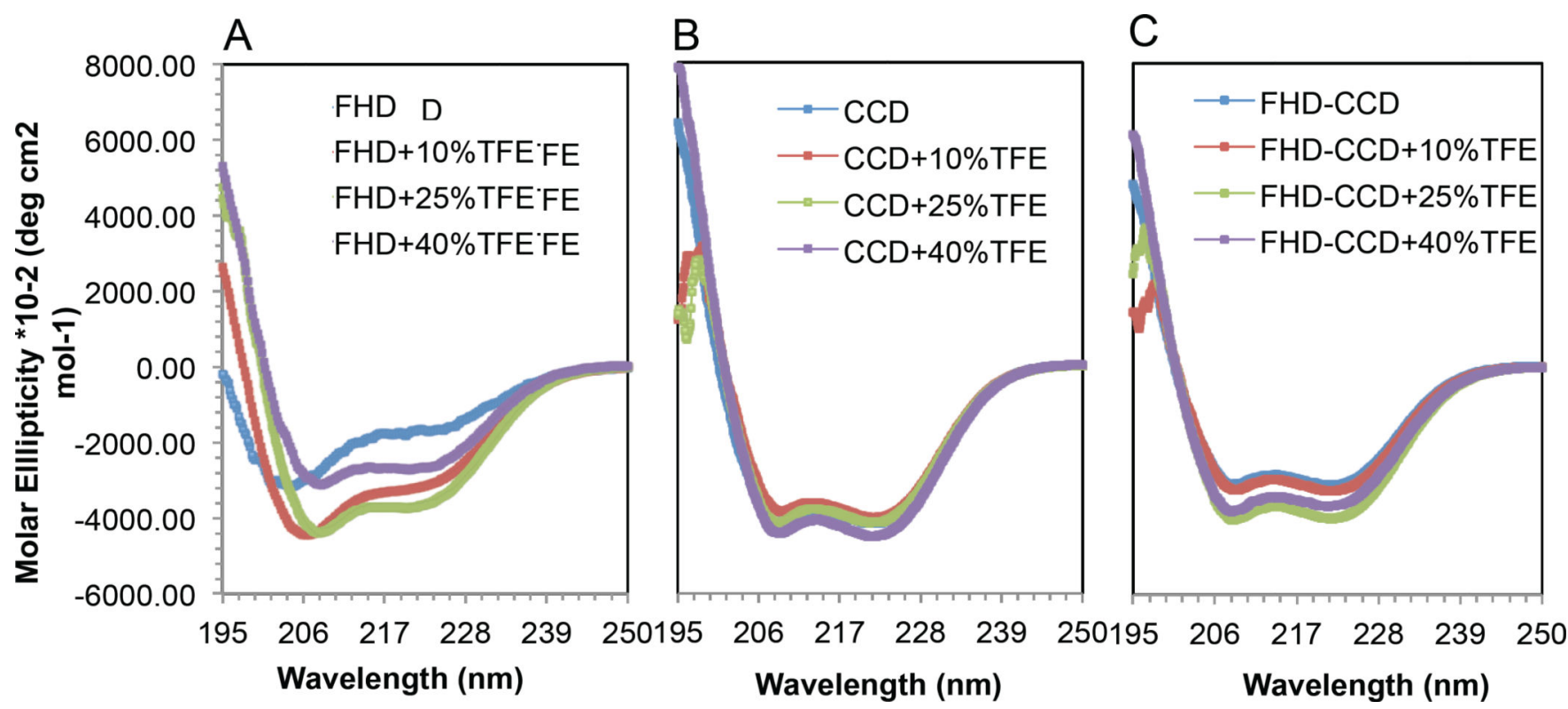

Figure 7.

Effects of TFE on the secondary structure content in various BECN1 constructs. CD spectra for the (A) FHD, (B) CCD and (C) FHD-CCD recorded at four different TFE v/v concentrations: $0 \%, 10 \%, 25 \%$ and $40 \%$. 
A

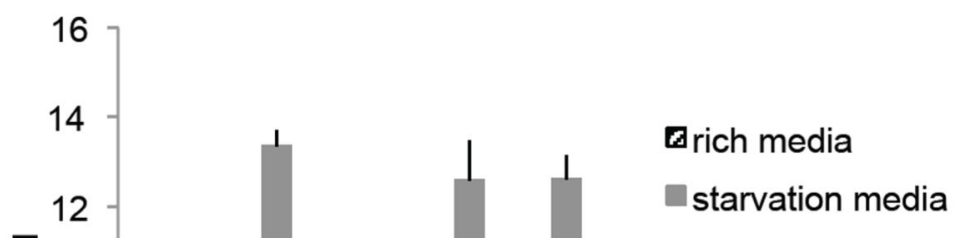

B

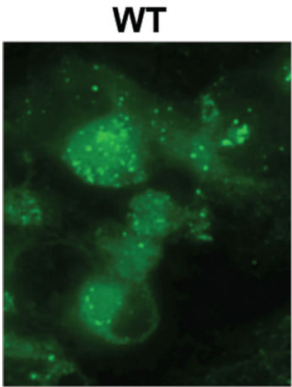

L144A

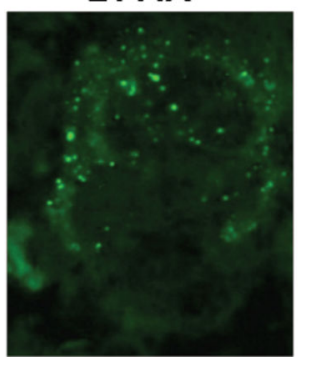

L148A

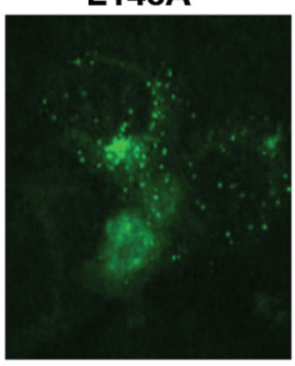

L152A

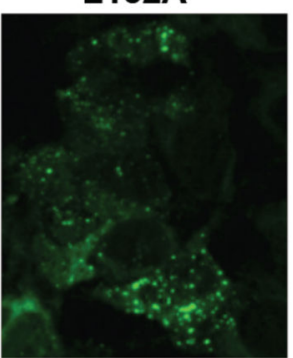

E158A

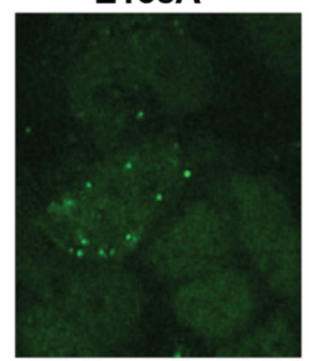

Y162A

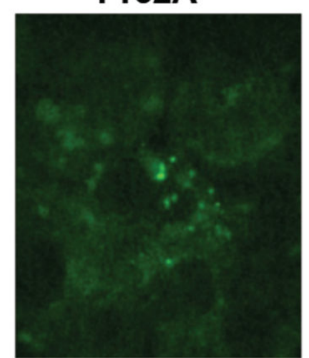

L166A

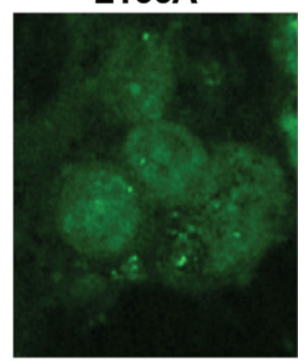

L169A

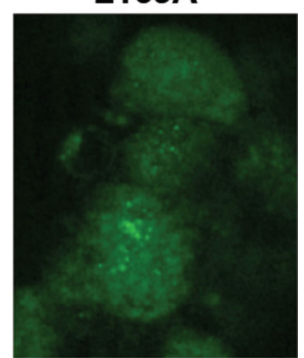

Figure 8.

Effect of the mutation of conserved BECN1 residues on autophagy. (A) Light microscopy quantification of discrete GFP-LC3 puncta per cell in GFP-positive MCF7 cells cotransfected with GFP-LC3, WT or mutant BECN1 as indicated below the X-axis. Bars represent number of puncta per cell for each construct. (B) Representative images of GFPLC3 staining in cells grown in starvation media and transfected with mutant BECN1 as indicated. 


\section{Table 1}

X-ray Diffraction Data Collection and Refinement Statistics ${ }^{a}$

\begin{tabular}{|c|c|}
\hline Data collection & \\
\hline Wavelength ( $\mathrm{A})$ & 1.3776 \\
\hline Data range $(\AA)$ & $44.66-1.95$ \\
\hline Space group & $\mathrm{P}_{3} 32$ \\
\hline Unit cell parameters & $a=b=c=63.16 \AA$ \\
\hline Mosaicity $\left(^{\circ}\right)$ & $0.093-0.200$ \\
\hline Unique reflections & 3514 \\
\hline Multiplicity & $75.5(78.1)$ \\
\hline Completeness (\%) & $100(100)$ \\
\hline${ }^{a} \mathrm{R}_{\text {sym }}(\%) \ddagger$ & $0.16(0.52)$ \\
\hline $\mathrm{I} / \sigma_{\mathrm{I}}$ & $26.3(1.8)$ \\
\hline \multicolumn{2}{|l|}{ Refinement } \\
\hline Model: & PDB ID: 5EFM \\
\hline Residues & 15 \\
\hline Water molecules & 8 \\
\hline Sulfate molecules & 1 \\
\hline Data Range $(\AA)$ & $44.66-1.95$ \\
\hline$b_{\mathrm{R}_{\mathrm{work}}}(\%)$ & 21.2 \\
\hline$b, c_{\mathrm{R}_{\text {free }}}(\%)$ & 21.6 \\
\hline \multicolumn{2}{|l|}{ Average B-values $\left(\AA^{2}\right)$} \\
\hline Main Chain & 46.4 \\
\hline Side Chain & 61.4 \\
\hline Water & 54.6 \\
\hline All Atoms & 54.5 \\
\hline \multicolumn{2}{|c|}{ B-factor RMSDs between bonded atoms $\left(\AA^{2}\right)$} \\
\hline Main chain & 5.722 \\
\hline Side Chain & 11.287 \\
\hline \multicolumn{2}{|l|}{ RMSDs from target values: } \\
\hline Bond Lengths $(\AA)$ & 0.009 \\
\hline Bond Angles $\left({ }^{\circ}\right)$ & 1.298 \\
\hline Ramachandran outliers & 0 \\
\hline
\end{tabular}

Values in parentheses pertain to the highest resolution shell.

${ }^{a} \mathrm{R}_{\mathrm{sym}}=\sum_{\mathrm{h}, \mathrm{i}}\left|\mathrm{I}_{\mathrm{h}, \mathrm{i}}-\mathrm{I}_{\mathrm{h}}>\right| / \sum_{\mathrm{h}, \mathrm{i}} \mathrm{I}_{\mathrm{h}, \mathrm{i}}$.

$b_{\mathrm{R} \text { factor }}=\sum \mathrm{h},\left|\mathrm{F}_{\text {obs }}-\right| \mathrm{F}_{\text {calc }}\left|/ \sum \mathrm{h}\right| \mathrm{F}_{\text {obs }} \mid$.

${ }^{c}$ Test set for $\mathrm{R}_{\text {free }}$ consisted of $5.5 \%$ of data. 
Table 2

$\mathrm{CD}$ analysis of secondary structure content of BECN1 constructs

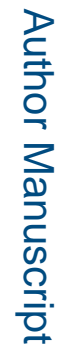

\begin{tabular}{|l|l|l|l|l|}
\hline \multirow{2}{*}{ Domain(s) } & \multirow{2}{*}{$\begin{array}{l}\text { Length } \\
\text { (residues) }\end{array}$} & \multicolumn{3}{|l|}{ \% Secondary Structure Estimation } \\
\cline { 3 - 5 } & & Helix & Sheet & Coil \\
\hline BH3D & 26 & 4 & 3.8 & 92 \\
\hline FHD & 31 & 37.8 & 17.8 & 49.4 \\
\hline CCD & 97 & 81.6 & 0.9 & 20.5 \\
\hline FHD-CCD & 128 & 80.6 & 0.2 & 20 \\
\hline BH3D-FHD-CCD & 164 & 64.4 & 5.2 & 34.6 \\
\hline
\end{tabular}

